\title{
Output Measurements of a Highly Overmoded W-band Source
}

\author{
A. J. MacLachlan, A. R. Phipps C. W. Robertson, H. Yin, L. Zhang, A. W. Cross, and A. D. R. Phelps \\ Department of Physics, SUPA, University of Strathclyde, Glasgow, G4 0NG, Scotland, UK \\ a.d.r.phelps@strath.ac.uk
}

\begin{abstract}
Use of overmoded interaction structures to obtain higher powers from mm-wave vacuum electronic sources is an approach that is being used in the research reported here. To provide mode selectivity a two dimensional corrugated periodic structure is constructed on the inner surface of the conducting cylindrical slow wave interaction region that is excited by an annular electron beam. This two dimensional structure consists of a periodic surface lattice that is designed to facilitate coupling of the wave fields to generate a powerful single frequency output, even though the ratio of the diameter of the structure to the emitted wavelength is $\sim 5$. Recent measurements of the output of this mm-wave source are presented.
\end{abstract}

\section{INTRODUCTION}

Two dimensional (2D) corrugated periodic structures have found many uses in microwave and mm-wave source research. They can be used as mode-selective Bragg mirrors in fast-wave devices, such as free electron lasers [1-3]. 2D corrugated periodic structures having a very similar structural appearance can be used in a rather different physical role to form a slow-wave interaction structure in which mode-selectivity is again featured but in overmoded Cherenkov microwave and mm-wave sources.

A research project to explore methods of increasing the power output from vacuum electronic mm-wave sources has been underway for some time at the University of Strathclyde [4-8]. One promising approach has been to increase the potential power output by increasing the crosssection of the interaction structure in which the electron beam transfers power to the electromagnetic wave field. This allows a larger beam current to be employed, which provided the efficiency can be maintained at reasonable levels implies greater mm-wave output power should result.

Normally increasing the cross-section of the interaction structure leads to a complex excitation of multiple modes and low efficiency of operation. Theoretical analysis [4-7] shows that by careful design of a periodic surface lattice (PSL) situated on the inner surface of the cylindrical electron beam - wave interaction structure can result in a coupling of surface waves and volume waves in the interaction structure to produce a single frequency powerful output mm-wave.

Results of an initial experiment have been published [9] and this presentation reports significant improvements that have been made during the present second experimental campaign.

\section{EXPERIMENT}

By corrugating the internal surface of the cylindrical interaction region with a carefully designed co-sinusoidal periodic corrugation, both in the axial direction and in the azimuthal direction, theoretical analysis predicts a rarefied output mode spectrum can be obtained even though a large number of modes can potentially be excited in a cylindrical structure of diameter $\mathrm{D}$, at a working wavelength $\lambda$, when $\mathrm{D} / \lambda \sim 5$, as in the present highly overmoded structure.

A cylindrical 2D PSL structure, of the type shown in Fig (1) can be described by equation (1)

$$
r=r_{0}+\Delta r \cos \left(k_{z} z\right) \cos (m \emptyset)
$$

where $r_{0}$ is the radius of the unperturbed waveguide, $\Delta r$ is the amplitude of the corrugation, $k_{\mathrm{z}}=2 \pi / d_{z}$, where $d_{z}$ is the period of the corrugation along the axial $\mathrm{z}$ - coordinate and $m$ is the number of azimuthal variations of the corrugation.

These 2D PSL structures have been manufactured using two different processes. A fairly conventional process of electrodeposition of copper on a carefully machined aluminium former, that is then dissolved away using strong alkali has been used successfully, as well as a 3D printing method [9].

In the initial experiment the electron beam was generated from a cold velvet cathode, as shown in Fig 2(a). whereas in the present experiments an improved knife-edge annular graphite cathode, as shown in Fig. 2(b), is used to produce a better-defined annular [10] electron beam. An axially-uniform DC magnet solenoid is used to guide the electron beam through the 2D cylindrical PSL interaction structure.

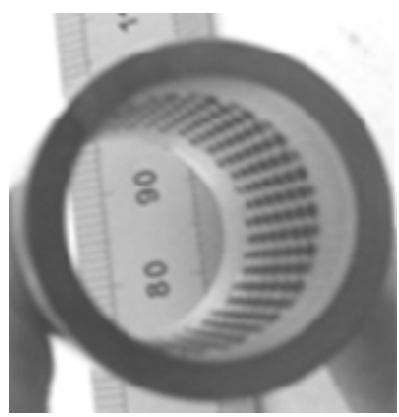

Figure 1. Example of highly overmoded cylindrical interaction structure with $2 \mathrm{D}$ periodic surface lattice on the internal surface of the cylinder. 


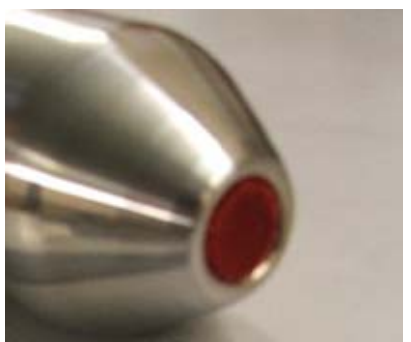

(a)

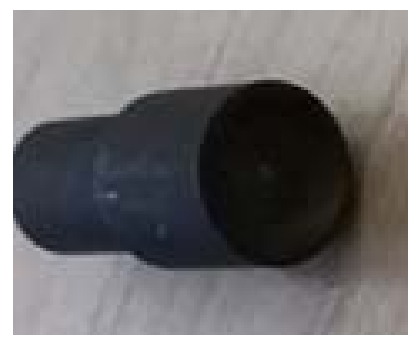

(b)
Figure 2. (a) Velvet cathode used in preliminary experiment (b) annular knife-edge graphite cathode being used in the present experiments.

In the initial experiments the output frequency was estimated by measuring the magnitude of the output after passing the output through a series of cut-off filters. In the more recent experiments the use of a W-band local oscillator and mixer in a heterodyne frequency measurement diagnostic allows a more precise determination of the output frequency.

\section{NUMERICAL MODELLING}

To compare with the laboratory experimental measurements a series of cases with initial parameters chosen to match those of the experiment have been numerically modelled using the CST software suite. The parameters and dimensions of the 2D PSL configuration, the radial position of the annular electron beam, its accelerating potential and current have been used as inputs to the numerical model. The results of the modelling show that, as expected, such a highly overmoded interaction structure is sensitive to relatively small changes in the input parameters. However outputs in the W-band frequency range with over $10 \%$ electronic efficiency are predicted by the modelling with appropriately chosen values of the length of the 2D PSL interaction region, with the optimum selected 2D PSL parameters $\mathrm{r}_{0}, \Delta \mathrm{r}, \mathrm{dz}, \mathrm{m}$, combined with the electron beam accelerating potentials and currents corresponding to those in the laboratory experiment.

\section{CONCLUSIONS}

Measurements of the output from an overmoded W-band slow wave source that uses a $2 \mathrm{D}$ periodic surface lattice in an upgraded experiment are reported. Compared with the initial experiment, the present experiment has a more nearly annular electron beam, the guiding solenoidal field is more uniform axially and the frequency measurement diagnostic enables a higher precision to be achieved. Output predictions obtained from numerical modelling using the CST software suite are also reported. Although this highly overmoded source shows greater sensitivity to variations in the input parameters, compared with a source operating on a low order mode, it is possible to achieve significant levels of electronic efficiency even when $\mathrm{D} / \lambda \sim 5$.

\section{ACKNOWLEDGMENT}

David Barclay is thanked for his skilled technical assistance. Ivan Konoplev is thanked for many helpful discussions. AFOSR provided support under award numbers FA8655-13-1-2132 and FA9550-17-1-0095. This research was carried out in collaboration with the FY'12 AFOSR Transformational Electromagnetics MURI and the Leverhulme Trust Network "Advanced Research on Generation of THz and X-ray Radiation” (IN-2015-012).

\section{REFERENCES}

[1] N. S. Ginzburg, N. Yu. Peskov, A. S. Sergeev, et al, "Mechanism of azimuthal mode selection in two-dimensional coaxial Bragg resonators", J. Appl. Phys., vol. 105, art. no. 124519, July 2009.

[2] N. S. Ginzburg, N. Yu. Peskov, A. S. Sergeev, et al., "Theory of free-electron maser with two-dimensional distributed feedback driven by an annular electron beam", J. Appl. Phys., vol. 92, pp. 1619-1629, Aug. 2002.

[3] N. S. Ginzburg, et al., "The use of a hybrid resonator consisting of one-dimensional and two-dimensional Bragg reflectors for generation of spatially coherent radiation in a coaxial free-electron laser," Phys. Plasmas, vol. 9, pp. 2798-2802, June 2002.

[4] I. V. Konoplev, et al., "Surface wave Cherenkov maser based on a periodic lattice", Appl. Phys. Lett., vol. 96, art. no. 261101, June 2010.

[5] I. V. Konoplev, et al., "Excitation of surface field cavity and coherence of electromagnetic field scattering on two-dimensional cylindrical lattice," Appl. Phys. Lett., vol.97, art. no. 261102, Dec. 2010 .

[6] I. V. Konoplev, et al., "Cylindrical, periodic surface lattice - Theory, dispersion analysis and experiment", Appl. Phys. Lett., vol. 101, art. no. 121111 , Sept. 2012.

[7] I. V. Konoplev, et al., "Cylindrical periodic surface lattice as a metadielectric: concept of a surface-field Cherenkov source of coherent radiation", Phys. Rev. A, vol. 84, art. no. 013826, July 2011.

[8] I. V. Konoplev, et al., "Surface field excitation by an obliquely incident wave", Appl. Phys. Lett., vol. 102, art. no. 141106, Apr. 2013.

[9] A. R. Phipps, A. J. MacLachlan, C. W. Robertson, et al., "Electron beam excitation of coherent sub-terahertz radiation in periodic structures manufactured by 3D printing," Nucl. Instrum. Methods Phys. Res. B, vol. 402, pp. 202-205, July 2017.

[10] I. V. Konoplev, et al., "High-current oversized annular electron beam formation for high-power microwave research," Appl. Phys. Lett., vol. 89 , art. no. 171503 , Oct. 2006. 